EOMmun: Communication et organisation

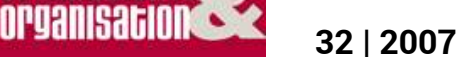

La ville dans tous les sens

\title{
Voiles et dévoilements du Havre
}

Béatrice Galinon-Mélénec

\section{(2) OpenEdition}

Journals

Édition électronique

URL : http://journals.openedition.org/communicationorganisation/310

DOI : 10.4000/communicationorganisation.310

ISSN : 1775-3546

\section{Éditeur}

Presses universitaires de Bordeaux

\section{Édition imprimée}

Date de publication : 1 décembre 2007

Pagination : 64-79

ISSN : 1168-5549

\section{Référence électronique}

Béatrice Galinon-Mélénec, « Voiles et dévoilements du Havre », Communication et organisation [En ligne], 32 | 2007, mis en ligne le 01 décembre 2010, consulté le 01 mai 2019. URL : http:// journals.openedition.org/communicationorganisation/310; DOI : 10.4000/ communicationorganisation.310 
Dossier : La ville dans tous les sens

\title{
Résumé
}

L'auteur expose ici comment deux organisations, la mairie du Havre et l'UNESCO, émettrices de messages destinés pour la première aux habitants de la ville et pour la seconde au «monde », ont mis en oeuvre des processus de reconstruction de représentation de cette ville. Ces deux écritures correspondant à deux registres de la reconnaissance, l'analogie et l'expertise, se sont conjuguées, pour faire effectivement évoluer la lecture de la ville du Havre.

\section{Mots-clés}

Mairie, Stratégies de communication, Evolution des représentations.

\begin{abstract}
The author explains how two institutions, two official bodies, namely the City of Le Havre and UNESCO, have generated messages to the people of Le Havre for the former, to the "world at large » for the latter and how both of them have implemented reconstruction processes of representation of the city. These two discourses corresponding to two levels of recognition, analogy and expertise, have combined effectively to alter the way the city of Le Havre is now perceived.
\end{abstract}

\section{Key-Words}

City Hall, communication Strategies, perception evolutions.

Béatrice Galinon-Mélénec est Professeur des universités en Sciences de l'Information et de la Communication et directrice du CDHET (Communication et Développement des Hommes, des Entreprises et des Territoires) réseau interdisciplinaire de chercheurs dont le siège se situe à l'Université du Havre (Département Information et Communication). 


\section{Voiles et dévoilements du Havre Béatrice Galinon-Mélénec}

galinon@free.fr

Dans le cadre d'une revue scientifique spécialisée dans la communication des organisations, il nous a tout d'abord semblé intéressant de présenter la campagne de communication d'une Mairie souhaitant faire évoluer les représentations des habitants de sa ville. Dans cet objectif, nous avons travaillé sur la démarche de l'émetteur (la Mairie du Havre) visant des destinataires (les habitants) au moyen d'une campagne d'affichage. Pour comprendre sa stratégie de communication, nous avons eu recours à l'examen de la revue $L E$ HAVRE OCEANES de 1999 à 2007, aux documents internes que le service de la communication du Havre a bien voulu nous fournir et à des entretiens. Pour situer le contexte d'émission, nous avons étudié les différents ouvrages cités en bibliographie. Sans vouloir trop simplifier la complexité de l'identité de la ville, il nous a semblé possible de s'appuyer sur les trois items les plus souvent énoncés : Le Havre, métropole portuaire; Le Havre, ville détruite en 1944, Le Havre, une architecture de reconstruction. Les conditions de production ainsi définies permettent de cerner d'emblée les limites de cette présentation. Elles ne nous paraissent pas mériter de renoncer à l'exposition des données ainsi recueillies, lesquelles peuvent servir de point de départ à une recherche plus approfondie; ce que nous appelons de nos vœux.

Le Havre est avant tout une grande ville portuaire et décline, à ce titre, toutes les caractéristiques de cette catégorie urbanistique. Nous ne développerons pas ce point qui, tout en étant essentiel, sort de notre propos. Nous préciserons seulement que le centre ville est proche de la mer, ce qui renforce d'une part l'identité maritime et d'autre part a obligé la ville à se développer vers les terres, au-delà des falaises. Or, le « centre » aujourd'hui ainsi « excentré » est précisément celui qui a été détruit. Le « cœur» de la ville ne correspond ni à la caractéristique géographique de «centre» ni à la lisibilité de la tradition. On ne s'étonnera donc pas d'observer que cette ville a tendance à être happée par un seul sens, celui de son histoire récente tragique. Cela lui confère une "substance » qui a tendu longtemps à l'éloigner des mirages de «La société du spectacle » (Debord).

Après avoir analysé nos sources, nous en sommes venus à moduler notre objectif initial et à examiner comment deux acteurs ont récemment participé à un déplacement du regard: "en interne» la 
Mairie ; en externe, l'UNESCO. Etant donné que le registre socio affectif est répandu dans les discours que les Havrais tiennent sur leur ville, notre propos tentera de rendre cette dimension « palpable».

\section{Le Havre, toutes voiles dehors : un port dans la ville}

"Il ressort d'une étude sur la presse nationale (Sorgem, 1997-2001) que l'image du Havre à travers les médias présente en premier item le Port $»^{1}$.

Dans cette ville détruite qu'ils ne reconnaissent plus, les Havrais s'accrochent encore et toujours à leur identité maritime : ils ne se lassent pas d'observer les entrées et sorties du port des porteconteneurs, sorte d'immeubles mobiles, qui lorsqu'ils apparaissent entre les tours de la Porte Océane semblent vouloir les dominer de leur imposante hauteur. À ces figures du dynamisme de la filière du transport des marchandises par voie maritime s'associent celles plus festives des paquebots et des voiliers. Les activités maritimes du Havre sont sa fierté. Les événements maritimes se succèdent renforçant leur image identitaire: Le France choisissant Le Havre comme port d'attache, les ACH (Ateliers et Chantiers du Havre) lancent (1985) le Wind Star - le plus grand paquebot à voile du monde -, la Transat J. Vabre y prend son départ depuis 1993. Ces événements sont relayés par la presse nationale dont les titres, par exemple Le Havre. La mer est son métier ${ }^{2}$, confortent sur le plan national et international la représentation maritime de la ville.

Et puis, il y a les docks. S'y chargent et s'y déchargent les marchandises $\mathrm{du}$ bout du monde dans une ambiance tellement particulière que les cinéastes en ont relayé, film après film, une image si forte que celle-ci en vient à dominer les autres dans les représentations de toute une génération de français cinéphiles ${ }^{3}$. "Quai des brumes ». Le titre seul du film de Marcel Carné (tourné en 19364) suffit à évoquer toute l'ambiance d'un port noyé dans le mystère. Les films noirs s'y lovent parfaitement; autant que les scènes d'amour romantiques. Plus de cinquante films ont été tournés au Havre. Ils ont ancré dans les représentations collectives l'idée que le Havre est lieu de trafic, une métropole maritime, où se croisent des populations de

\footnotetext{
${ }^{1}$ Jean-René Martel, directeur de la communication de la Mairie du Havre, 2001.

${ }^{2}$ Le Point, octobre 2003, ${ }^{\circ} 1624$.

${ }^{3}$ La réponse généralisée des Français à la question «que représente pour vous le Havre? » («Un grand port») trouve une partie non négligeable de sa source dans l'impact du registre cinématographique sur l'imaginaire collectif.

${ }^{4}$ Cette année-là, l'ouragan qui traverse la ville à $235 \mathrm{~km} /$ heure rappelle une fois de plus à la France la situation géographique de la ville.
} 
tous les milieux, venues de tous les coins du monde dans des perspectives très diverses aussi bien commerciales que touristiques ou même militaires. Le Havre, Ville Port, induit dans l'imaginaire collectif les rêves ou les cauchemars que cette taxinomie engendre. Le Havre, comme toute ville portuaire, est d'abord un "espace d'émotions ${ }^{5} \gg$.

\section{Le voile du deuil : la destruction du Havre}

Pour les Havrais de souche, le 5 septembre 1944 est un jour qui domine tous les autres ${ }^{6}$. Ce jour-là, les raids alliés détruisent le centre ville du Havre. La ville dont les Havrais étaient si fiers est réduite à l'état de poussière. Le film, Table Rase (Christian Zarifian, 1988)', donne à voir à tous ceux qui ne l'auraient pas vécue l'ampleur du traumatisme ${ }^{8}$. Le trauma est tel qu'année après année, et malgré tous les efforts de reconstruction urbaine, ce qui dominera ce sera la déconstruction identitaire. Car, en bombardant la ville, les alliés ont détruit bien plus que des bâtiments; ils ont tué bien plus que des civils, des hommes et des femmes en détresse sous l'occupation, ils ont détruit l'âme de la ville, les traces de l'histoire, et puis plus grave encore, la confiance. Dès lors, les Havrais déjà endurcis par une nature maritime qui impose sa loi se replient sur eux-mêmes. Ils se méfient des «nouveaux arrivants", assument une image de "taiseux» et quand ils brisent le silence, c'est pour inlassablement évoquer leur ville « d'avant ».

Le Havre, à l'embouchure de la Seine, a abandonné la brique, le charme des colombages, la chaleur du bois, pour lui préférer... le

\footnotetext{
${ }^{5}$ Alain Leménorel in Mériel 0., Leménorel A., Le Havre entre réel et imaginaire, Cabourg, 2005. p. 5.

${ }^{6}$ A la question «Quel est l'événement ou le souvenir qui vous a le plus marqué » posée aux lecteurs de la revue Océanes, la réponse la plus fréquente est «La période de guerre, les bombardements, les privations et la destruction de l'église Saint Joseph le 5 septembre $1944 »$ (décembre 1999).

${ }^{7}$ L'ambiance dramatique de la ville détruite se retrouve également dans Un homme marche dans la ville de Marcel Pagliero (1949), film d'ailleurs insupportable pour ceux qui ont souffert dans le plus intime d'eux-mêmes et qui y voient une intolérable caricature.

${ }^{8}$ Présenté en septembre 2007 au programme du Studio dans les termes suivants: «(...) 3000 civils sous les bombes de la RAF. Et un long et lourd silence, et beaucoup de honte autour de cet événement dont personne ne sait que faire, qu'il vaudrait mieux oublier si on pouvait, s'il ne continuait pas à agiter les consciences sourdement. Tel est le sujet profond de Table Rase... ».
} 
béton'. Jusqu'à peu, quand le touriste faisait halte et interrogeait les habitants, il s'entendait simplement rétorquer: «ce n'est pas un choix », signifiant ainsi que ce béton lui est étranger. Pire, il le rejette. Il est le signe trace de la blessure toujours ouverte : la destruction du Havre par les alliés. Ici, "Le souvenir des morts en est le béton» (Pierre Jouve). Ici se révèle en permanence la relation plus ou moins exprimée entre mémoire et territoires.

\section{Perret parie sur l'avenir : le choix d'une architecture moderne}

"Ce que je veux, c'est faire quelque chose de neuf et de durable. Puisque nous sommes à zéro, il faut en profiter pour partir sur des bases nouvelles qui permettront de faire face à l'avenir de grande ville et de grand port que Le Havre a devant lui ${ }^{10}$ " (Auguste Perret, 1945).

Quand, en 1945, Perret est chargé de reconstruire le centre du Havre, c'est avec soin qu'il pense à l'avenir des Havrais. Il conçoit une métropole du futur tournée à la fois vers la mer et vers la terre. Il veut que les habitants profitent pleinement de l'espace, de la lumière et de l'air. Il imagine des appartements que la lumière envahit. N'est-ce pas la lumière du ciel havrais qui a attiré tant de peintres? Le monde entier ne connaît-il pas la façon dont Claude Monet a fixé sur la toile les lumières de la «Porte Océane » du Havre dans Impression soleil levant?

Et le choix du matériau? Un matériau moderne, le béton. Apte à défier le temps, il peut faire face aux pires assauts des éléments naturels maritimes, le vent et l'eau. Perret souhaite que tout soit fonctionnel et économique ("L'économie c'est la chasteté de l'art» dit-il dans une Conférence radiophonique du 17 février 1939). Aller à l'essentiel. Pas d'ornements superflus. "C'est dans la splendeur du vrai que l'édifice atteint la beauté ${ }^{11}{ }^{\prime}$.

Et les rues? Larges, longues, aux croisements orthogonaux ${ }^{12}$, elles permettront le développement de la circulation des véhicules qui ne manquera pas de s'opérer avec la croissance de la ville.

\footnotetext{
${ }^{9}$ Auguste Perret expérimenta l'utilisation du béton dès 1899 pour construire le Casino de Saint Malo (aujourd'hui détruit), testant ainsi une nouvelle conception de l'architecture.

${ }^{10}$ Duteurtre Vincent, En pleine lumière, LE HAVRE, Paris, Gallimard, 2006, p. 11.

${ }^{11}$ Auguste Perret in Contribution à une théorie de l'architecture, cité par Danielle Knapp, p. 10-4.

${ }^{12}$ La trame initiale (1541) du plan de la ville du Havre, déjà orthogonale, était le fruit de l'ingénieur J. Bellamarto.
} 
Tout cela est vrai. Mais rien n'y fait. Là où Perret imaginait la fluidité de la circulation, les Havrais voient un manque de vie. Là où, il bâtissait du solide, ils voient de la froideur. Cette architecture soustraite au hasard, donc à l'inconnu, devrait apaiser les peurs. Elle apparaît sans surprise, sans mystère, austère, triste. Certains la nomment «Stalingrad-sur-mer ${ }^{13}$ ». L'espace bâti est un langage nonverbal ; la relation entre cet espace et les habitants, une interaction que les habitants intériorisent. Il faudrait prendre le temps de l'expliciter. Faute d'en disposer dans ce cadre, nous renvoyons le lecteur aux ouvrages des sémioticiens de la ville.

Les Havrais n'aiment pas leur ville et, quand les hebdomadaires, l'Express et Le Point, année après année, situent le Havre dans les dernières places des villes "Où il fait bon vivre en France », ces médias contribuent, non seulement à créer ou renforcer la représentation nationale que « les Français » ont de la ville, mais aussi celle des habitants eux-mêmes. N'ayant pas fini leur travail de deuil, les Havrais ne sont pas en mesure de la défendre. Ils «se retrouvent dans un environnement (...) dans lequel leur mode de vie a perdu son sens, dans les rues qu'ils ne reconnaissent pas, où le familier a fait place à l'inconnu » (Marchand, 2001).

Le pari du modernisme n'est pas pour autant abandonné par ceux qui pensent l'avenir de la ville. Le 18 novembre 1982, l'espace Oscar Niemeyer, le grand centre culturel du Havre, est inauguré. Oscar Niemeyer et Auguste Perret, bâtisseurs du cœur du Havre. Ces deux architectes ont marqué le $\mathrm{XX}^{\mathrm{e}}$ siècle, avec Le Corbusier... Trois grands architectes, trois grandes villes - Brasilia, Chandigarh, Le Havre - qui symbolisent l'architecture spécifique du $\mathrm{XX}^{\mathrm{e}}$ siècle.

En 2007, le musée Malraux ${ }^{14}$ du Havre se fait l'écho de ce rapprochement en réunissant les œuvres de dix photographes et d'un vidéaste $^{15}$. Tout en faisant la part belle aux peintres révélant les jeux de lumières de la Normandie ${ }^{16}$ côté mer ou côté campagne ou à ceux que Le Havre a formés (exposition Jean Dubuffet, 2001), ce musée a entrepris depuis longtemps de former l'œil des Havrais à des formes

\footnotetext{
${ }^{13}$ Citation de D. Morrison in Financial Times, septembre 2006.

${ }^{14}$ Initialement «musée-maison de la culture », inauguré (1961) par André Malraux, il marque lui aussi l'architecture havraise non seulement par son style résolument moderne qui joue sur la transparence et la lumière mais aussi par son exceptionnel emplacement de bord de mer.

${ }^{15}$ Annette Haudiquet, conservateur du Musée Malraux, in le catalogue de l'exposition Brasilia, Chandigarh, Le Havre,(2007), p. 11-14.

${ }^{16}$ Gestes, signes, traces, espaces, exposition du musée Malraux, février-avril 2007.
} 
d'expression artistique plus diversifiées. Cependant, étant donné que les jugements de goût et les classifications sociologiques présentées par Pierre Bourdieu dans La distinction, critique sociale du jugement (1979) n'ont pas ici perdu de leur pertinence, on ne s'étonnera pas de trouver une corrélation entre distribution de capital culturel et de capital économique et les différentes «dispositions » au regard de l'art en général et de l'avant-garde en particulier.

Petit à petit, une petite fraction de la population, les intellectuels goûtant la modernité, fait entendre sa voix. Ils propagent des discours « savants » sur l'architecture Perret et sur la modernité havraise. Ils attirent ceux qui veulent participer à cette démarche initiatique, à titre de « producteur» ou de « consommateur». Il devient de bon ton dans une certaine « avant-garde » de comprendre «la poétique du béton ${ }^{17}$ » et de s'inscrire dans un mouvement qui valorise le nouveau rapport architecture-ville-urbanisme/ mode de vie des habitants.

La ville attire les artistes contemporains et plus seulement, dans la lignée des impressionnistes, les peintres mais aussi les photographes ${ }^{18}$ toujours fascinés par ses étranges lumières changeantes qui transforment totalement le paysage selon l'heure où on le regarde.

L'espace Oscar Niemeyer, surnommé «Le Volcan» est non seulement une figure de l'architecture havraise, il est aussi un haut lieu d'expression culturelle. Le Volcan soutient le métissage des pratiques sociologiques ${ }^{19}$ et vise à brouiller la relation «bourdieusienne» qui «veut» que «les répartitions de capital économique et de capital culturel cloisonnent les pratiques et les choix esthétiques ». Il met en place des expositions destinées aux «RMISTES ». Il facilite l'expression de l'imaginaire de la rue. L'exemple le plus spectaculaire est le Géant $(1993)^{20}$ de la compagnie Royal de Luxe. Très apprécié, il entraîne à sa suite tous les Havrais, sans distinction de milieu, qui se rassemblent en masse pour le suivre

\footnotetext{
${ }^{17}$ Nous voudrions signaler ici la construction de la superbe « Cité de la musique » (en cours de construction en 2007). Façonnée en béton, elle a été conçue par Christian de Portzamparc pour Rio de Janeiro (Exposition Portzamparc, Rêver la ville, Cité de l'architecture et du patrimoine, mars-septembre 2007).

${ }^{18} \mathrm{Cf}$. bibliographie.

${ }^{19}$ Le Volcan cherche à rassembler toutes les formes de culture et s'adresse à tous les publics, comme en témoigne sa "Découverte du spectacle vivant et du milieu artistique » à destination des personnes titulaires du RMI. Il participe ainsi à une des images du Havre: «Le Havre, une ville qui a du cœur», Le Havre Océanes, mai 2000 , p. 21. Les différents indicateurs, dont se fait l'écho la presse nationale, mettent en évidence l'importance de l'entraide et de l'assistance dans cette ville.

${ }^{20}$ Marionnette géante d'une extraordinaire expressivité.
} 
en une procession admirative dans toutes les rues de la ville. Derrière l'apparence de la fête, se joue un événement majeur pour la ville : l'appropriation de l'espace reconstruit en un espace qui se vit joyeusement et poétiquement, car la mise en scène de ce Géant emporte vers le rêve tous les spectateurs du plus petit au plus grand.

Ces deux lieux culturels havrais associent donc au modernisme du contenant le dynamisme et l'innovation du contenu. Forme et fond associés dans un même objectif : relever Le Havre de ses blessures, le rendre plus solide et plus rayonnant qu'avant sa destruction, faire de ses différences internes et externes une force d'évolution. Leurs initiatives amorcent une évolution, préparent le terrain à la future « révélation» que constituera plus tard le classement de l'UNESCO. Leur action n'est pas pour autant déterminante. Au moment de leur création, les évènements qu'ils organisent ne suffisent pas pour que «les Havrais soient fiers de leur ville».

Le goût de la modernité reste encore longtemps le choix de quelquesuns réussissant à convaincre les plus modernes, le plus souvent «nouveaux arrivants $»^{21}$, ou une fraction de la population havraise qui n'a pas connu la guerre. Restait donc à réconcilier l'ensemble des Havrais avec leur ville. 50 ans de deuil (1944-1994). La durée étaitelle suffisante pour espérer redonner le goût de leur ville aux Havrais, ces hommes et femmes éloignés de tous les vains artifices?

C'est sur ce thème de campagne ("Redonner aux Havrais la fierté de leur ville ») qu'un homme de communication, Antoine Rufenacht, fut élu maire de la ville du Havre en juin 1995. L'équipe de la communication conduite par Jean-René Martel $^{22}$ prépara et mit en place une première campagne de communication en 1998. Plusieurs vagues d'affichage ont eu lieu. Il n'y a pas eu d'études d'impact. Pour estimer l'écho auprès des destinataires, nous avons été contraints de nous référer à un élément quantitatif : les affiches aussitôt sorties de presse furent aussitôt distribuées en réponse à la demande des commerçants et des particuliers. À la recherche de ces affiches en vue de cette publication, nous avons nous-même cherché plusieurs semaines avant de pouvoir obtenir directement du service de la

\footnotetext{
${ }^{21}$ Ainsi dès 2002, Danielle Knapp, arrivée il y a peu au Havre, apporte aux Havrais un regard neuf sur l'œuvre d'Auguste Perret et de Marguerite Hué (elle a créé les verrières de l'église Saint Joseph), dans Auguste Perret et Marguerite Hure, jusqu'à la sereine délectation. Elle écrit (p. 1-9) concernant l'église "J'ai envie de faire partager ce que j'ai trouvé : un vertige esthétique et spirituel".

${ }^{22}$ Avec lequel nous nous sommes entretenus pour reconstituer la logique des plans de communication qu'il a orchestrés.
} 
communication les documents internes (textes et visuels) qui ont guidé l'organisation de cette campagne d'affichage. C'est une rapide synthèse des informations ainsi recueillies que nous proposons ici.

\section{Vers la reconstruction identitaire : le rôle de la communication}

La première campagne affiche son souhait de transformer le regard des Havrais sur leur ville. Une observation d'avant campagne permettra de repérer que ce regard n'était pas aussi homogène qu'il y parait au premier abord ${ }^{23}$ : il diffère selon les groupes sociaux et leurs schèmes de classification; les quartiers apparaissent cloisonnés : les habitants circulent peu d'un quartier à un autre :'il n'y a pas un «Le Havre» mais plusieurs «Le Havre». À partir de ce constat, la campagne va chercher à faire évoluer les regards vers une reconnaissance mutuelle. En s'appuyant sur des registres connus (les sites célèbres du monde entier, des images transversales aux différents groupes sociaux), elle va chercher à provoquer un accord général : «Notre ville, quartier par quartier, est une belle ville, digne d'être vue».

La première série d'affiches présente une photo et une question. Les Havrais sont ainsi conviés, sous forme de jeu, à identifier les lieux de leur ville qui ont été photographiés. Ce choix du questionnement pour déplacer le regard est, avec le dévoilement de la réponse le vecteur d'un sentiment fort d'identification et de fierté. Et cela d'autant plus, qu'il s'agit bien de photos de la ville telles que chaque havrais peut, en se situant au même endroit, la saisir en utilisant un appareil photographique simple. Dans la mesure où par un commentaire, l'image renvoie à des espaces sosies qui engendrent le rêve, il se crée une représentation entre "réel et imaginaire » pour reprendre le titre du très beau livre d'Olivier Mériel et Alain Lemenorel ${ }^{24}$.

Cette première campagne d'affichage, intitulée Ma ville aujourd'hui, avait pour vocation de resituer géographiquement et dans la mémoire commune un fourmillement de détails issus du patrimoine architectural havrais. Le message était simple : «regardez le Havre d'aujourd'hui; vous pouvez être fier de votre ville $»^{25}$. En termes d'analyse, nous dirons que le choix de l'image fixe a produit une force de sens et que le commentaire associé à l'image restreint les

\footnotetext{
${ }^{23}$ Il y a toujours eu la ville d'en haut et la ville d'en bas, mais c'était «Deux corps, une âme ; un seul et même effort, un seul et même essor» (René Coty).

${ }^{24}$ Mériel 0., Leménorel A., Le Havre entre réel et imaginaire, Cabourg, Editions Cahiers du temps, 2005.

${ }^{25}$ Source : la direction de la communication de la Mairie du Havre.
} 
de sens et que le commentaire associé à l'image restreint les possibilités interprétatives $\mathrm{du}$ spectateur. Chaque quartier ainsi valorisé, affiché dans tous les autres quartiers de la ville, invite au voyage. Le simple fait de regarder les affiches permet de cheminer en rêve dans cette ville aux multiples visages. L'imaginaire de la ville se décloisonne.

Beaucoup de Havrais, très portés pour des causes historiques à la méfiance vis-à-vis de l'étrangéité, se sentaient plus ou moins assignés à résidence dans leur quartier. Ces images d'invitation au voyage dans les différents «mondes » que constituent les quartiers transforment radicalement le rapport du Havrais à sa ville.

Cette campagne constitue un marquage temporel dans l'histoire des représentations du Havre. Les Havrais entament à partir de cette campagne un processus d'appropriation positive de l'environnement direct, quartier par quartier.

Nous fournissons ici quelques exemples des commentaires associés aux photographies qui ont été diffusées sous forme d'affiches de 2 et 8 $\mathrm{m}^{2}$ dans tout le Havre. 17 visuels commentés représentaient les 17 quartiers du Havre.

\section{Une ville mosaïque qui comprend toutes les autres villes}

Les photos offertes au regard visaient à « révéler» l'espace urbain en créant un nouvel "entrelacs entre l'expérience quotidienne de la ville, ses matérialités et l'imaginaire ${ }^{26} »$.

Le commentaire des photos, quartier par quartier, pourrait contribuer à renforcer l'idée d'une ville fractionnée en territoires. Mais, le choix d'une esthétique généralisée renvoyant à une même fiction, "l'ailleurs » avec son prestige, contribue au contraire à homogénéiser la représentation : l'unité recherchée n'est pas architecturale, elle est affective.

La volonté affirmée est de connoter positivement l'environnement. Les désordres de la ville, qui existent comme dans toute métropole, même non détruite par la guerre, ne sont pas mis en scène. Il ne s'agit pas ici comme dans une certaine photographie contemporaine de se faire l'écho visuel des déchets ou de la désolation de certains lieux ${ }^{27}$. Les photographies ne prétendent pas à la création artistique. Elles et

\footnotetext{
${ }^{26}$ Expression d'Alain Leménorel, citée hors contexte.

${ }^{27}$ Mons A., L'ombre de la Ville, essai sur la photographie urbaine contemporaine, éditions de la Villette, 1994.
} 
leurs commentaires visent un objectif précis: donner une vision positive de la ville, déplacer le regard pour faire prendre conscience qu'existent dans les détails de chaque quartier, même le plus démuni, une poésie qui ne demande qu'à être découverte.

Certes, la promenade de bord de plage n'a pas attendu les photographes pour rappeler les Havrais aux plaisirs des sens. Marcher le long de la plage, sentir le vent balayer son visage, entendre le chant des mouettes et des goélands, compagnons familiers de la promenade, regarder les mouvements des piétons, se fondre dans la foule qui se regroupe rituellement pour la sortie dominicale et sentir son corps vibrer de tous ses sens dans la force des éléments naturels. Aussi n'est ce pas cette part de la ville que la campagne de communication révèle.

Mettre en lumière la beauté cachée des lieux «incompris» ou partiellement compris et mettre en place un système d'échoïsation des quartiers via des photographies révélant le plein existentiel de chaque quartier, tisser un nouveau réseau de significations, voilà la raison de la conduite de ce projet communicationnel.

\section{Le dévoilement de la valeur du patrimoine architectural}

Restait la zone du centre ville détruite en 1944. Reconstruite d'après le plan d'une équipe dirigé par l'architecte Auguste Perret, elle n'avait pas conquis le cœur des Havrais. Le temps passant (50 ans), il fallait envisager la maintenance des bâtiments. Pouvait-on envisager d'associer les Havrais à sa conservation et à sa restauration ? Ce fut là la deuxième étape clef de l'effort de communication pour faire évoluer les représentations des Havrais. La connaissance de la valeur de l'architecture Perret a accompagné cette démarche.

Là encore, le choix de la connotation est poétique ("Perret et la poétique du béton", exposition du Musée Malraux). Le terme semblait a priori osé. Un oxymore même pour certains Havrais. N'est-ce pas plutôt respecter profondément la pensée de Perret qui définissait l'architecte comme "un poète qui pense et qui parle en construction $»^{28}$.

Les publications se multiplient, les expositions aussi. L'exposition Perret "La poétique du béton" circule, d'abord à Paris, puis dans d'autres villes du monde. Les Havrais comprennent progressivement que ces édifices modernes qui symbolisent à leurs yeux la destruction de 1944, symbolisent pour d'autres une compétence particulière, très

\footnotetext{
${ }^{28}$ Perret A., Techniques et Architecture, tome 2, 1942.
} 
recherchée : la compétence d'édifier ${ }^{29}$.

En 2001, La ville obtient le label "ville d'art et d'histoire". C'est une première étape. Mais, c'est en 2005 quand l'UNESCO inscrit cette architecture sur la liste du Patrimoine de l'Humanité qu'est réellement mis en évidence qu'il convient d'être fier de cet "exemple remarquable de l'architecture et l'urbanisme de l'après-guerre, fondé sur l'unité de méthodologie et le recours à la préfabrication, l'utilisation systématique d'une trame modulaire, et l'exploitation novatrice du potentiel du béton ».

Une étape nouvelle est alors franchie dans le dévoilement de l'intérêt de cette ville. Les Français dans leur ensemble comprennent que le Havre n'est pas seulement une grande métropole portuaire, le premier port conteneurs de France, son deuxième port pétrolier, mais un lieu incontournable de visite au même titre que le Mont-Saint-Michel, la cathédrale de Chartres, le palais et le parc de Versailles, le centre historique d'Avignon ou de Lyon, autant de sites français classés au Patrimoine Mondial de l'UNESCO.

Il ne s'agit plus de valoriser les lieux par une campagne de communication interne à la ville. Il s'agit d'une reconnaissance internationale. Les Havrais qui soignaient leurs cicatrices et se repliaient sur eux-mêmes s'ouvrent désormais à l'environnement humain et non humain. De plus en plus aptes à saisir les opportunités que leur offre une situation de choix (la mer dans une ville qui a la dimension et l'activité d'une métropole), ils pratiquent dans l'enthousiasme d'une dynamique nouvelle qui la natation, qui la planche à voile, qui le kitesurf, qui le skate-board, qui le roller, qui le vélo, qui la voile, qui le golf, qui le foot, qui l'aviron, qui la danse, qui... La liste est trop longue pour en faire ici l'inventaire.

Côté mer, le Havre n'est plus seulement une métropole portuaire, c'est aussi une ville de sports et de loisirs ${ }^{30}$. Le terre-plein de la plage aménagé dans les années 60, le port de plaisance dans les années 70 , le front de mer dans les années 90 mettent en valeur le littoral. Les labels (pavillon bleu) attestent de sa dimension balnéaire. Les Havrais, en toutes saisons - et il y a les quatre saisons chaque jour de l'année dans cette ville qui vit au rythme des marées et des vents qui amènent et chassent les nuages plusieurs fois par jour - profitent de «leur mer» dans la ville.

\footnotetext{
${ }^{29}$ Choay F., L'allégorie du patrimoine, Paris, Seuil - 1999.

${ }^{30}$ Item $n^{\circ} 3$ de l'enquête SORGEM déjà citée.
} 
Dossier : La ville dans tous les sens

\section{Conclusion}

Difficile de modifier les représentations qui tirent une grande part dans la "mémoire à long terme ». De «mémoire d'homme», au Havre, elle est cicatricielle. La date du 5 septembre 1944, sans cesse ravivée par les récits de ceux qui l'ont vécue et par ceux qui au travers de films et de documentaires se soumettent au "devoir de mémoire », demeure LA date identitaire. Les représentations liées à l'exécution quotidienne d'actions ${ }^{31}$ jouent certes aussi, dans la " mémoire à court terme ». Comment un Havrais n'aurait-il pas intégré que se déplacer en voiture au centre du Havre ne pose aucune difficulté ? Reposant en grande partie sur la sensori-motricité, la mémoire immédiate ne résiste pas à l'expérience. La "représentation imagée », celle que nous avons exposée ici à propos de la campagne de communication de la Mairie du Havre, pose plus de difficulté ; car, que voit-on quand on regarde ? On voit des «signes» que l'on nomme «immeuble», «arbre, «bateau», utilisant ainsi le registre lexical et les taxinomies généralement admises et socialement transmises. Le jugement de goût sur «l'objet» intervient dès lors que l'objet perçu reçoit une qualification ( « beau », "vilain», etc.). Ce jugement semble a priori strictement individuel. Néanmoins les sociologues ont depuis longtemps démontré que des régularités s'observaient et que des regroupements par "famille de goûts » permettaient d'établir un lien entre conditions de vie, histoire de vie, expérience de vie - autant de termes relativement voisins - et pratiques, en particulier évaluatives. Chercher à faire évoluer les représentations de plusieurs groupes sociaux pour les faire converger suppose donc de trouver un critère d'évaluation transversalement reconnu. Cela revient à rechercher "le plus petit dénominateur»- ici en termes de capacité à "rendre légitime » une image positive du Havre - à ces différents groupes. À partir des observations retranscrites ici, nous pouvons conclure que la légitimation de la rupture architecturale que constitue la reconstruction Perret est encore en devenir en ce qui concerne sa généralisation à tous les groupes sociaux. Comme toute innovation, sa signification ne peut être d'emblée reconnue par l'ensemble de la population. Ce qui est, dommageable dans la mesure où "nous sommes le paysage où nous vivons, cela forme notre conscience » Bates, 1992 $2^{32}$.

\footnotetext{
${ }^{31}$ Pour plus de précisions sur ces distinctions, lire Ghiglione R, Bonnet C., Richard J.F., Traité de psychologie cognitive, Paris Dunod, (3 tomes), 1990.

${ }^{32}$ Cité par D. Marchand, 2001.
} 
- Par l'association de représentations imagées (les photos de détails de la ville) et de représentations propositionnelles (les commentaires) à des " ailleurs » connotés positivement par le plus grand nombre. En ce sens nous dirons que le service de communication de la Mairie du Havre s'est appuyé sur un processus de reconnaissance analogique.

- Par l'entrée dans une classification mondialement reconnue (celle de l'UNESCO) : il s'agit ici d'un processus de reconnaissance par l'expertise. En effet, en intégrant dans la catégorie "Patrimoine de l'humanité » l'invention architecturale d'Auguste Perret, l'UNESCO a certes d'abord provoqué une forme de dissonance avec l'auto-perception de la majorité des Havrais (et de nombreux Français). Mais la consistance cognitive d'«évaluateurs éclairés» que représente l'expertise de l'UNESCO a conduit les plus aptes à la contemporanéité de la reconnaissance à situer les édifices $\mathrm{du} \mathrm{XX}^{\mathrm{e}}$ siècle dans une perspective historique.

- Par le rôle joué par les médias nationaux ${ }^{33}$ dont on connait le pouvoir de légitimation dans le « grand public ».

- En assurant la diffusion médiatique de l'événement (la reconnaissance patrimoniale de la valeur de l'architecture Perret).

- En opérant un "véritable basculement» des classements opérés sur la liste ordonnée des "villes où il fait bon vivre en France ", « une sorte d'avant-après » la reconnaissance par l'UNESCO, ils ont largement contribué, si ce n'est à modifier radicalement les représentations, du moins à introduire une brèche dans les représentations négatives de la ville. Ainsi, Donald Morrison in le Financial Times de septembre 2006: "l'année dernière, j'ai lu que l'UNESCO venait d'inscrire Le Havre au patrimoine mondial. Non, vous ne rêvez pas. (...). À l'évidence, une nouvelle visite s'imposait (...) et mes yeux se sont dessillés. Car si cette ville est en effet une citadelle en béton, elle est aussi un petit paradis tant pour ses habitants que pour ses visiteurs $»$.

Nous ne saurions prétendre avoir présenté la ville du Havre «dans tous les sens ». Nous espérons seulement, à l'occasion de cette publication, avoir levé des voiles, non pas ceux qui enveloppent la

\footnotetext{
${ }^{33} 500$ articles dans la presse nationale et internationale évoquant le classement du Havre par l'UNESCO (Vincent Duteurtre).
} 
Dossier : La ville dans tous les sens

ville de subtiles lumières, mais ceux qui, épaissis du poids du passé, obscurcissent le regard.

\section{Bibliographie}

Barthes R., "Sémiologie et urbanisme" in : L'aventure sémiologique, Paris, Editions du Seuil, 1985.

Bevort A., Dezalay T., Guessaz A., Eliot E., Maillet C., Yon A., $L a$ construction du rapport aux services collectifs dans un espace local. Représentations de l'espace-temps, pratiques d'accès, comportements de mobilité des usagers et rôle du tissu associatif (Le Havre, Rouen), Le Havre, CIRTAI, 2004.

Bourdieu P., La distinction, critique sociale $d u$ jugement, Paris, Editions de minuit, 1979

Brasilia, Chandigarh, Le Havre, Catalogue de l'exposition du musée Malraux, Le Havre, juin-septembre 2007.

Brocard M., La perception des identités urbaines, Le Havre, Editions CIRTAI, 2000.

Certeau M. (de), L'invention au quotidien, vol 1, Paris, Edition UGE, 1980.

Choay F., L'allégorie du patrimoine, Paris, Editions du Seuil, 1999.

Dezalay T., Gueissaz A., Elio E., Développement et diversification de l'offre culturelle: Enquête auprès des structures et des professionnels, Le Havre, Editions CIRTAI, 2004.

Ducruet C., Les villes-ports, laboratoires de la mondialisation, Thèse, Le Havre, décembre 2004.

Duteurtre V., En pleine lumière, LE HAVRE, Paris, Editions Gallimard, 2006.

Fremont A., Les stratégies des armateurs de lignes régulières en matière de dessertes maritimes, Le Havre, Editions CIRTAI, 2006.

Gestes, signes, traces, espaces, Catalogue de l'exposition du musée Malraux, Le Havre, février-avril 2007.

Ghiglione R., Bonnet C., Richard J.F., Traité de psychologie cognitive, Paris, Editions Dunod, (3 tomes), 1990.

Jouve P., Le Havre/Ville port, Paris, Editions Isthme, 2005.

Knapp D., Guérin R., Auguste Perret Marguerite Hure, jusqu'à la sereine délectation, Darnétal, Editions Petit à petit, 2002. 
Lanta M.C., Guillaume H., Marca R., Le Havre des petits bonheurs, Paris, Editions Le lou du lac, 2004.

Le magazine mensuel de la ville du Havre Le Havre Océanes, tous les $\mathrm{n}^{\circ}$ de 1999 à nos jours et, son prédécesseur, CITE LE HAVRE n 95, " Mémoires vivantes 1944-1994 ", Juin 1994.

Lecanu G., Mémoires en image, Le Havre, Saint-Cyr-sur-Loire, Editions Alan Sutton, 1995.

Leveque L., La transformation des espaces urbanisés dans l'aire métropolitaine Caen, Rouen, Le Havre, Le Havre, Editions CIRTAI, 1999.

Marchand D., Relations entre la structure urbaine, les modes d'appropriation et les représentations conceptuelles de la ville, Thèse de doctorat, Paris V, 2001.

Marchand D., Représentation de la ville du Havre, Mémoire de DEA, EHESS, 1994.

Mériel 0., Leménorel A., Le Havre entre réel et imaginaire, Cabourg, 2005.

Mons A., L'ombre de la ville, essai sur la photographie urbaine contemporaine, Editions de la Villette, 1994.

Morrisson D., "Le Havre, paradis en béton armé" in: Financial Times, Londres, retranscrit in Courrier International, septembre 2006.

Plans des campagnes de communication (documents internes au service) de la Mairie du Havre.

Travaux des chercheurs du Centre Interdisciplinaire de Recherche en Transports et Affaires Internationales (CIRTAI), Université du Havre 